\section{P26 Food-Related Decision Patterns Among SNAP Participants in Mississippi}

Virginia Gray,PhD, RDN, virginia.gray@csulb.edu, California State University, Long Beach, 1250 Bellflower Blvd, Long Beach, CA 90840; Alisha Hardman, PhD, CFLE, Mississippi State University; Sylvia Byrd, PhD, LD, RDN, Mississippi State University

Background: While food assistance programs in the US have collectively shifted from a primary focus on food provision to include an emphasis on diet quality, insight from program participants may shed light on ways to improve nutritional impacts.

Objective: The objective of the current study was to qualitatively explore food-related decision patterns among SNAP recipients in Mississippi.

Study Design, Settings, Participants: Focus groups $(\mathrm{n}=18)$ were conducted with 127 low-income female caregivers of children under the age of 18. The Health Belief Model was used as a guide to assess food-related decision patterns. Cluster sampling was used to randomly recruit participants from the four regional divisions of Mississippi State University Extension for participation in the study.

Measurable Outcome/Analysis: Audio recorded focus groups were transcribed verbatim and coded by two independent researchers using thematic analysis.

Results: Drivers of food selection among participants often overlapped with barriers to healthy eating. Participants used many strategies to manage food costs and viewed healthy foods as out of reach and quick to perish. Cost, taste preferences, habits, and lifestyle/family factors were primary drivers of food selection and preparation; each of these areas presented barriers to healthy eating. Health issues were most often shared as a driver of food selection once disease was established. Participants reported a variety of strategies used in striving for healthier eating.

Conclusion: SNAP participants in Mississippi report a variety of strategies for managing food costs and striving for healthier eating, while also reporting barriers. Focusing on ways to manage costs of healthy foods, honor taste preferences, and work within lifestyle/family contexts may enhance efforts to support healthy eating in this audience.

Funding: SNAP-Ed.

\section{P27 Fruit and Vegetable Recipes Promoted at a Mobile Farmers' Market in a Food Desert in the District of Columbia Ward 8}

Tiffany Johnson-Largent, PhD, LD, RDN, tiffany. johnsonlarge@udc.edu, University of the District of Columbia, College of Agriculture, Urban Sustainability, and Environmental Sciences, 4200 Connecticut Ave, NW, Rm 117, Washington, DC 20008; Lillie Monroe-Lord, PhD, $L D, R D N$, University of the District of Columbia, College of Agriculture, Urban Sustainability, and Environmental Sciences; Elgloria Harrison, DM, RRT, AE-C, College of Agriculture, Urban Sustainability, and Environmental Sciences

Background: Nutritional-related diseases such as hypertension and diabetes continue unabated in the District of
Columbia Ward 8 whose residents are primarily African Americans. Wards are administrative divisions with approximately 75,000 persons. The District of Columbia Behavioral Risk Factor Surveillance System (BRFSS) Annual Health Report confirmed that fruit and vegetable (FV) consumption were low among African Americans, especially in Ward 8 (Anderson-Morgan, Fett, Jasso, Moten, \& Harrison, 2018) May - November 2018 a mobile Farmers' Market was located at the Bellevue Library to provide the residents with access to fresh FV. Recipe tastings and demonstrations were included to demonstrate the ease of preparing produce that was purchased from the Farmers' Market. Six recipes were introduced: pasta veggie salad; 3bean salad; radish tomato salsa; summer squash salad; tangy savory slaw, and mint citrus water. Data was collected to determine whether residents planned to prepare these recipes at home.

Objective: Three main objectives of this research were: a) determine the frequency of consumption of $\mathrm{FV}$; b) determine the ease of recipe preparation, and c) determine improvement in the health and nutritional status of the residents.

Study Design, Settings, Participants: A five-question survey was administered to 152 attendees at the Famers' Market located in Ward 8. Results for question two are included here "After tasting this recipe, do you plan to make it at home?"

Measurable Outcome/Analysis: Attendees who tasted recipes during this six-week period, 93\% planned to make these recipes at home.

Results: The results of attendees showed that 93\% said yes, $5 \%$ said no, $2 \%$ did not respond, and $1 \%$ said maybe.

Conclusion: Nutrition-education and recipes that are tasty and easy to make are key steps in changing behaviors of residents who are at high risk of nutritional-related diseases.

Funding: None.

\section{P28 Qualitative Study: User Experiences with Platano, a Dietary Self-Monitoring App for Underserved Patients with Type 2 Diabetes}

Filippa Juul, MSc, New York University; Lena Mamykina, PhD, Columbia University; Amenda Almonte, BA, Stony Brook University; Jhack Sepulveda, $B A, R D$, Columbia University; Elizabeth Heitkemper, PhD, RN, Columbia University; Elliot G. Mitchell, MA, Columbia University; Marissa Burgermaster,PhD, marissa.burgermaster@austin. utexas.edu, University of Texas at Austin, 1400 Barbara Jordan Blvd, Austin, TX 78723

Background: Dietary self-monitoring is a key strategy in diabetes self-management and may be facilitated by mHealth. However, smartphone applications are typically designed for high resource audiences and may exacerbate health inequity if they fail to meet user needs in underserved groups.

Objective: We aimed to understand user experiences with a dietary self-monitoring smartphone application 